\title{
E-vehicle Service Architecture for Logistic Systems
}

\author{
Sebastian Apel and Volkmar Schau
}

\begin{abstract}
Until the year 2020, Germany has established a national development plan with the goal to push one million fully electric vehicles into use. Part of the plan is to establish a number of federally funded research projects, which investigate and tackle domain specific problems, e.g. the limited driving range of electric cars. Freight traffic is especially hampered by those range restrictions. The Smart City Logistik project (www.armor.uni-jena.de/www.smartcitylogistik.de) strives for a practical and short-term solution to this problem in the concrete context set by the city of Erfurt, Germany. The focus is on ICT-support for currently available, small and medium sized, fully electric vehicles that provide for the "last mile" in freight handling. This poster provides the first results of on going work to construct an architecture managing these requirements with a special focus on how to handle the wide range of interfaces.
\end{abstract}

\footnotetext{
S. Apel $(\bowtie) \cdot$ V. Schau

Department of Computer Science, Friedrich Schiller University Jena,

07743 Jena, Germany

e-mail: sebastian.apel@uni-jena.de

V. Schau

e-mail: volkmar.schau@uni-jena.de 